\title{
A Projection Method for Closed-loop Identification
}

\author{
1997-09-17

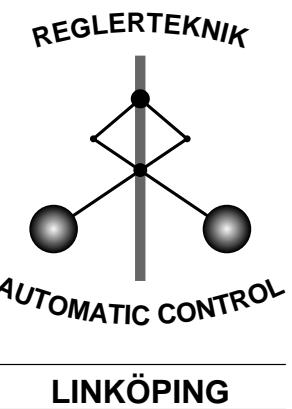

Urban Forssell and Lennart Ljung

Department of Electrical Engineering

Linkping University, S-581 83 Linkping, Sweden

WWW: http://www. control.isy.liu.se

Email: ufo@isy.liu.se, ljung@isy.liu.se

Report no.: LiTH-ISY-R-1984

Submitted to IEEE Transactions on Automatic Control

Technical reports from the Automatic Control group in Linköping are available by anonymous ftp at the address 130.236.20.24 (ftp. control.isy.liu.se). This report is contained in the compressed postscript file 1984 .ps.Z. 


\title{
A Projection Method for Closed-loop Identification
}

\author{
Urban Forssell and Lennart Ljung*
}

November 20, 1997

\begin{abstract}
A new method for closed-loop identification that allows fitting the model to the data with arbitrary frequency weighting is described and analyzed. Just as the direct method this new method is applicable to systems with arbitrary feedback mechanisms. This is in sharp contrast with other methods, such as the indirect method and the two-stage method, which assume linear feedback. To illustrate the effect of nonlinear elements in the feedback loop on these closed-loop identification methods a simulation study is presented.
\end{abstract}

\section{Introduction}

In "Identification for Control" the goal is to construct models that are suitable for control design. It is widely appreciated that small model uncertainty around the cross-over frequency is essential for successful control design. Consequently there has been a substantial interest in identification methods that provide a tunable optimality criterion so that the model can be fit to the data with a suitable frequency weighting. With open-loop experiments this is no problem: It is well known that arbitrary frequency weighting can be obtained by applying a prediction error method to an output error model structure with a suitable fixed noise model/prefilter (recall that the effect of any prefilter may be included in the noise model). However, open-loop experiments are not always possible since the system might be unstable or has to be controlled for safety or production economic reasons. In such cases closed-loop experiments have to be used. The problem is now that

${ }^{*}$ U. Forssell and L. Ljung are both with Department of Electrical Engineering, Linköping University, S-581 83 Linköping, Sweden. Email: ufo@isy.liu.se, ljung@isy.liu.se. the simple approach of using an output error model with a fixed noise model/prefilter will give biased results when applied directly to closed-loop data, unless the fixed noise model correctly models the true noise color (see, e.g., Theorem 8.3 in [4]). A way around this would be to use a sufficiently flexible parameterized noise model. This would eliminate the bias but the frequency weighting would then not be fixed.

In this contribution we describe and analyze a new closed-loop identification method that is consistent and, in the case of under-modeling, allows fitting the model to the data with arbitrary frequency weighting. The new method will be referred to as the projection method. The projection method is in form similar to the two-stage method [5] although the underlying ideas are different.

\section{Methods for Closed-loop Identification}

Depending on what assumptions are made on the feedback, one may distinguish between three different classes of closed-loop identification methods: $D i$ rect, indirect and joint input-output methods $[2,3]$. To limit the scope we will exclusively study methods derived in the prediction error framework.

In the direct method one applies a prediction error method to input-output data directly, ignoring possible feedback. Given that the noise properties are correctly modeled, the direct method gives consistent estimates of optimal accuracy. Since only inputoutput data is used, the direct method can be applied to systems with arbitrary feedback mechanisms. Another advantage of this method is that no special software is required. A drawback is that for consistency we need good, flexible (parameterized) noise models. Because of this it is not possible to fit the model to 
the data with a fixed, user-specified frequency domain weighting, as is possible in open loop with an output error-type model with fixed noise model.

The indirect methods give consistent estimates even with fixed noise models. The idea is to use the knowledge of the controller to transform the closedloop identification problem into an open-loop one. Consider the following set-up: The true system is

$$
\begin{aligned}
& y(t)=G_{0}(q) u(t)+v(t) \\
& v(t)=H_{0}(q) e(t)
\end{aligned}
$$

Here $e$ is white noise with variance $\lambda_{0}$. The regulator is

$$
u(t)=r(t)-F_{y}(q) y(t)
$$

The reference signal $r$ is assumed independent of the noise $e$. We also assume that the regulator stabilizes the system and that either $G_{0}$ or $F_{y}$ contains a delay so that the closed-loop system is well defined. The closed-loop system is

$$
G_{c l, 0}(q)=\frac{G_{0}(q)}{1+G_{0}(q) F_{y}(q)}=G_{0}(q) S_{0}(q)
$$

where $S_{0}$ is the sensitivity function,

$$
S_{0}(q)=\frac{1}{1+G_{0}(q) F_{y}(q)}
$$

The output can be written

$$
y(t)=G_{c l, 0}(q) r(t)+S_{0}(q) v(t)
$$

Now, the principle in all indirect methods is to identify the closed-loop system using measurements of $y$ and $r$ (note that this is an open-loop problem since $r$ and $e$ are uncorrelated) and then to compute an estimate of the open-loop system using the knowledge of the controller $F_{y}$. From (3) we see that the second step involves solving for $\hat{G}$ in

$$
\hat{G}_{c l}(q)=\frac{\hat{G}(q)}{1+\hat{G}(q) F_{y}(q)}
$$

Solving this equation will typically, due to numerical errors, lead to high-order estimates $\hat{G}$. However, help is not far away. Note that, using prediction error methods (that allow arbitrary parameterizations) this second step can be avoided if we parameterize the closed-loop model in terms of the open-loop model, that is, if we use a model of the following kind (see exercise $14 \mathrm{~T} .2$ in [4]):

$$
y(t)=\frac{G(q, \theta)}{1+G(q, \theta) F_{y}(q)} r(t)+H_{*}(q) e(t)
$$

Here $H_{*}$ is a fixed noise model. This special parameterization will be assumed in this paper.

The third class is the joint input-output methods. The basic idea in these methods is to model the input and output jointly as outputs from a system driven by the reference signal (and noise). Note that with the regulator given by (2), the input is given by

$$
u(t)=S_{0}(q) r(t)-F_{y}(q) S_{0}(q) v(t)
$$

so there is a close relationship between the output (5) and the input (8) which one can take advantage of. In the joint input-output methods no explicit knowledge of the controller is required except that it must be known to be of a certain (linear) form, for example the one in (2). This is in contrast with the indirect methods which require perfect knowledge of the regulator.

Here we will study a particularly interesting and robust joint input-output method called the two-stage method [5]. The two-stage method is usually presented using the following steps.

1. Identify the sensitivity function $S_{0}$ using measurements of $u$ and $r$ (cf. Equation (8)).

2. Construct the signal $\hat{u}=\hat{S} r$ and identify the open-loop system as the mapping from $\hat{u}$ to $y$.

This method deserves a couple of remarks. First, in the first step a high-order model of $S_{0}$ can be used since we in the second step can control the open-loop model order independently. Second, the simulated signal $\hat{u}$ will be the noise-free part of the input signal in the feedback system. Thus $\hat{u}$ clearly is independent of the noise $e$ and a fixed noise model/prefilter can be used to shape the bias.

The simplicity and robustness of the two-stage method makes it an attractive alternative for closedloop identification. To gain further insight in the properties of this method we now review some consistency results that was first given in [5]. 
Suppose that we from the first step have obtained an estimate $\hat{S}$ of the sensitivity function and we use a model of the following kind in the second step:

$$
y(t)=G(q, \theta) \hat{S}(q) r(t)+H_{*}(q) e(t)
$$

Here the noise model $H_{*}$ is fixed. From standard prediction error theory we then know that the limiting $G$-estimate will be characterized by (neglecting the arguments $\omega$ and $e^{i \omega}$ )

$$
G_{\text {opt }}=\arg \min _{G} \int_{-\pi}^{\pi}\left|G_{0} S_{0}-G \hat{S}\right|^{2} W d \omega, W=\frac{\Phi_{r}}{\left|H_{*}\right|^{2}}
$$

Note that

$$
\left|G_{0} S_{0}-G \hat{S}\right|^{2}=\left|\left(G_{0}-G\right) S_{0}+G\left(S_{0}-\hat{S}\right)\right|^{2}
$$

It is clear that for cases where $\hat{S} \neq S_{0}$ we will have a bias-pull towards models $G$ that minimize (11) $\hat{G}=G_{0}$ will not be optimal. However, if we in the first step have obtained a very accurate estimate of the sensitivity function $S_{0}$ this effect is negligible so that

$$
\left|G_{0} S_{0}-G \hat{S}\right|^{2} \approx\left|G_{0}-G\right|^{2}\left|S_{0}\right|^{2}
$$

In this case the mismatch $G_{0}-G$ will be minimized with a frequency weighting that is given by

$$
\bar{W}=\frac{\left|S_{0}\right|^{2} \Phi_{r}}{\left|H_{*}\right|^{2}}
$$

\section{The Projection Method}

We will now present the main contribution of this paper: The projection method. In form this method will be similar to the two-stage method but the motivation for the methods will be quite different.

The projection method can be understood as follows. Just as the two-stage method this method consists of two separate steps where in the first, preliminary, step we should "project" the input $\{u(t)\}$ onto the reference signal $\{r(t)\}$ using a noncausal, doubly infinite FIR-filter. This will result in a partitioning of the input signal into two orthogonal (uncorrelated) parts:

$$
u(t)=u_{\|}(t)+u_{\perp}(t)
$$

where

$$
u_{\|}(t)=S(q) r(t)=\lim _{M \rightarrow \infty} \sum_{k=-M}^{M} s_{k} r(t-k)
$$

Here it is assumed that $M$ does not grow faster than $N$ (the number of data), that is,

$$
M \rightarrow \infty, N \rightarrow \infty, \text { and } \frac{M}{N} \rightarrow 0
$$

The basic relation

$$
y(t)=G_{0}(q) u(t)+v(t)
$$

can thus be rewritten as

$$
\begin{aligned}
y(t) & =G_{0}(q) u_{\|}(t)+w(t) \\
w(t) & =G_{0}(q) u_{\perp}(t)+v(t)
\end{aligned}
$$

The point is now that, since $w$ is uncorrelated with $r$ and hence uncorrelated with $u_{\|}$, any identification method can be applied to the constructed inputoutput pair $\left\{u_{\|}, y\right\}$. We can for instance apply a prediction error method to the model

$$
y(t)=G(q, \theta) u_{\|}(t)+H_{*}(q) e(t)
$$

This would give the limiting estimate

$$
G_{o p t}=\arg \min _{G} \int_{-\pi}^{\pi}\left|G_{0}-G\right|^{2} W d \omega, W=\frac{\Phi_{u_{\|}}}{\left|H_{*}\right|^{2}}
$$

Here it is important to realize that the spectrum $\Phi_{u_{\|}}$ is known to the user when applying the second step of the algorithm. Hence, by properly choosing the noise model $H_{*}$, an arbitrary frequency weighting $W$ can be achieved.

From (18) it is also clear that for consistency of the projection method it is not required that the constructed signal $u_{\|}$equals the noise-free part of the input $u$, that is, $\hat{S}=S_{0}$ is not required, as is the case for the two-stage method (cf. Eq. (10)). A consequence of this is that the projection method can be applied to systems with arbitrary feedback mechanisms and still give consistent estimates, regardless of the noise model used. The price we pay is an increased variance error due to the extra noise term $G_{0} u_{\perp}$ in (16).

Before studying the variance properties of the projection method we would like to make the following additional remarks: 
- Here we chose to perform the projection using a noncausal FIR-filter but this step may also be performed non-parametrically as in Akaike's cross-spectral method [1].

- It would also be possible to project both the input $u$ and the output $y$ onto $r$ in the first step. This is in fact what Akaike suggested.

- As stated here, the formulas for the projection method coincide with those of the two-stage method except that $S$ should be parameterized in a different way.

- In practice $u_{\|}$will depend on the noise realization, since $u$ does. However, as this is a second order effect, the errors induced by ignoring this dependence will be neglected. Exact conditions for when it is safe to do so can be found in [6].

- Finally, in practice $M$ can be chosen rather small. Good results are often obtained even with modest values of $M$, see the simulation example below.

Let us now turn to the accuracy properties of this method.

Note that when deriving asymptotic variance expressions for the projection method we may use standard open-loop results for the asymptotic variance of the parameter estimates. From expression (9.55) in [4] we have

$$
\begin{aligned}
\operatorname{Cov} \hat{\theta} & \sim \frac{1}{N} R^{-1} Q R^{-1} \\
R & =\frac{1}{2 \pi} \int_{-\pi}^{\pi} \frac{\Phi_{u_{\|}}}{\left|H_{*}\right|^{2}} G_{\theta}^{\prime} G_{\theta}^{* *} d \omega \\
Q & =\frac{1}{2 \pi} \int_{-\pi}^{\pi} \frac{\Phi_{w} \Phi_{u_{\|}}}{\left|H_{*}\right|^{4}} G_{\theta}^{\prime} G_{\theta}^{*} d \omega
\end{aligned}
$$

Here $G_{\theta}^{\prime}$ denotes the gradient of $G$ taken w.r.t. $\theta$. It follows that the expression for the covariance is equivalent to the open-loop expression, except that $\Phi_{u}$ is replaced by $\Phi_{u_{\|}}$and $\Phi_{v}$ by $\Phi_{w}$. The partitioning (13) implies that

$$
\Phi_{u_{\|}}=\Phi_{u}-\Phi_{u_{\perp}}=\Phi_{u}\left[1-\frac{\Phi_{u_{\perp}}}{\Phi_{u}}\right]
$$

Thus, whenever $\Phi_{u_{\perp}} \neq 0$,

$$
\Phi_{u_{\|}}<\Phi_{u}
$$

Furthermore, in most practical cases we will also have

$$
\Phi_{w}>\Phi_{v}
$$

It is clear that for the projection method the signal to noise ratio will, in general, be worse than for open-loop identification $\left(\Phi_{u_{\|}} / \Phi_{w}<\Phi_{u} / \Phi_{v}\right)$, hence the variance will be larger.

\section{Simulation Example}

In this section we illustrate the performance of the closed-loop methods presented earlier when applied to a system with a nonlinearity in the loop.

In order to high-light the bias errors in the resulting estimates we performed a noise free simulation $(v \equiv$ 0 ) of the system depicted in Figure 1. The data was generated using a unit variance white noise reference signal $r$ and $N=500$ data samples were collected. The system $G_{0}$ is given by

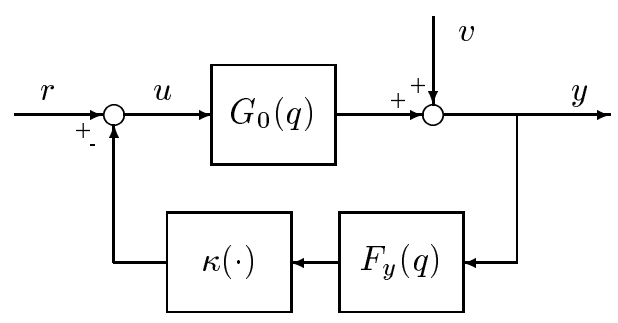

Figure 1: Closed-loop system with non-linear feedback.

$$
G_{0}(q)=\frac{b_{1} q^{-1}}{1+f_{1} q^{-1}+f_{2} q^{-2}}
$$

where $b_{1}=1, f_{1}=-1.2$, and $f_{2}=0.61$. The feedback controller is $F_{y}(q)=0.25$ and at the output of the controller there is a static nonlinear element $\kappa$ given by

$$
\kappa(x)= \begin{cases}x+3, & \text { if } x>0 \\ x-3, & \text { if } x<0\end{cases}
$$


The goal is to identify the open-loop system $G_{0}$ using the simulated closed-loop data. Four different methods will be considered: The direct, the indirect, the two-stage, and the projection method. These methods will not be further presented in this section. For technical details about the implementation of these methods the reader is referred to the descriptions of the methods given in the preceeding sections.

In the indirect and two-stage methods we will proceed as if the nonlinearity was not present, that is, we will assume that the closed-loop system is given by

$$
\begin{aligned}
& y(t)=G_{0}(q) S_{0}(q) r(t)+S_{0}(q) v(t) \\
& u(t)=S(q) r(t)-F_{y}(q) S_{0}(q) v(t)
\end{aligned}
$$

Note that the direct and projection methods require no knowledge/assumptions on the system to be applicable.

In the first step of the two-stage method we used a 10th order output error model and for the projection method the noncausal FIR filter had 41 taps (i.e., $M=20$ in Eq. (14)).

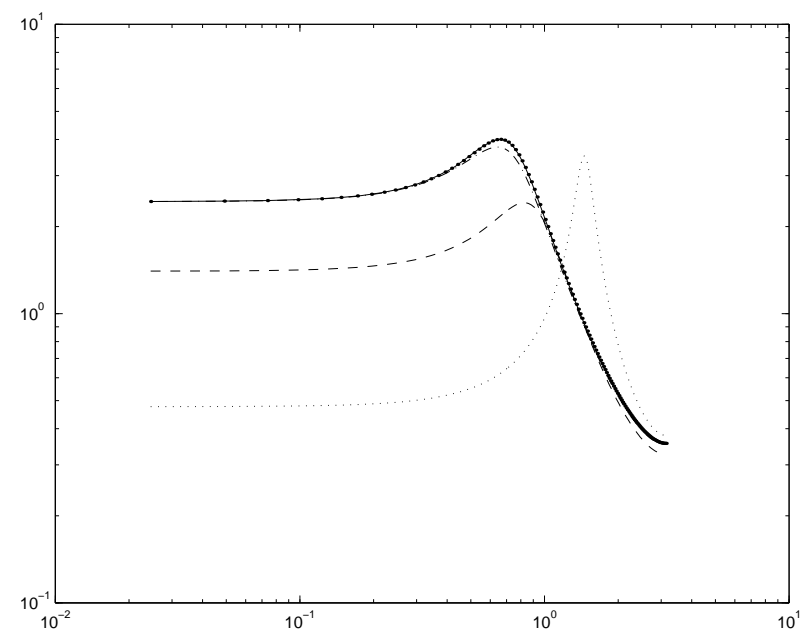

Figure 2: Bode plot of true and estimated transfer functions. Solid: True system; Point: Direct method; Dotted: Indirect method; Dashed: Twostage method; Dash-dotted: Projection method.

The result of the identification is shown in Figure 2. As can be seen the direct method gives a per- fect estimate of the true system. The indirect method performs very badly, which could be expected since there is a considerable error in the assumed feedback law. It is also clear that the projection method is able to model the true system quite accurately while the two-stage method performs less well. The performance of the projection method can be improved by increasing the parameter $M$ in the first-step model, here we quite arbitrarily chose $M=20$. For the twostage method, on the other hand, there is no clear relationship between the size of the first-step model and the final result. The identification results are summarized in Table 1.

Table 1: Summary of identification results. $(\mathrm{D}=$ Direct method; I= Indirect method; $\mathrm{T}=$ Two-stage method; $\mathrm{P}=$ Projection method)

\begin{tabular}{cccccc}
\hline & $\begin{array}{c}\text { True } \\
\text { value }\end{array}$ & $\mathrm{D}$ & $\mathrm{I}$ & $\mathrm{T}$ & $\mathrm{P}$ \\
\hline$b_{1}$ & 1.0000 & 1.0000 & 0.7536 & 0.8214 & 0.9790 \\
$f_{1}$ & -1.2000 & -1.2000 & -0.2034 & -0.9670 & -1.1884 \\
$f_{2}$ & 0.6100 & 0.6100 & 0.7842 & 0.5532 & 0.5900 \\
\hline
\end{tabular}

To further analyze why the projection method outperforms the two-stage method, which in form is quite similar to the projection method, we studied the impulse response coefficients of the first-step models in the two methods. The top plot in Figure 3 shows the estimated impulse response of the map from the reference $r$ to the input $u$. This impulse response was computed using correlation analysis. The middle and bottom plots show the impulse responses for the first-step models in the two-stage and projection methods, respectively. It is clear that the causal part of the impulse response is quite accurately modeled in the two-stage method while the noncausal part is zero. The noncausal FIR filter used in the projection method approximates the impulse response well for lags between the chosen limits -20 and 20. Thus, as this figure shows, the only principal difference between the two-stage and the projection methods is how the impulse response of the map from $r$ to $u$ is modeled - causally or noncausally. From this example it is also clear that, in general, the noncausal part of the impulse response can not be neglected without introducing errors in the resulting open-loop model. Note that this is true for nonlinear closed-loop systems. For linear systems the map from the reference 

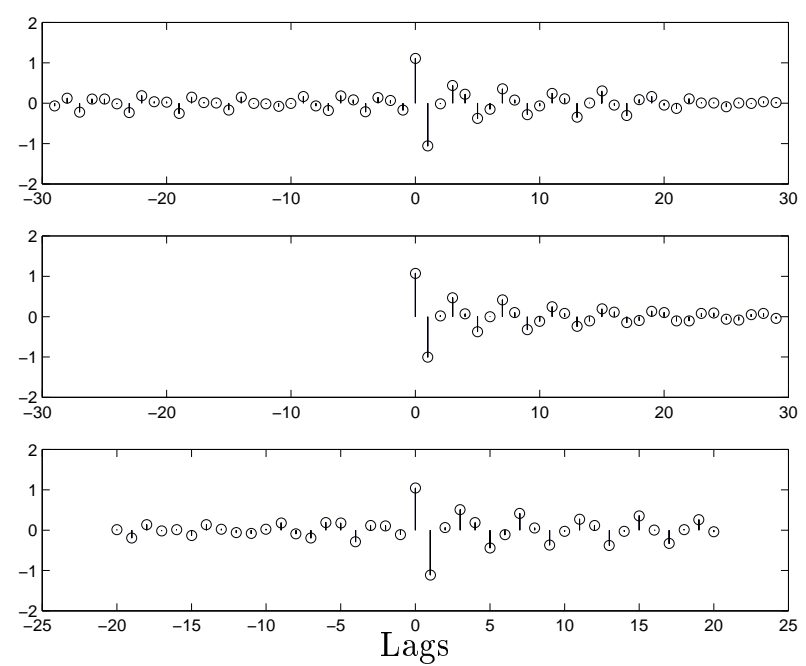

Figure 3: Estimated impulse response (top) and impulse responses for the first-step models in the twostage (middle) and the projection (bottom) methods.

$r$ to the input $u$ is the sensitivity function $S_{0}$ which is causal. In this case there will be no difference between the two-stage and the projection method, given, of course, that the whole causal impulse response is correctly modeled.

\section{Conclusions}

The direct method gives consistency and optimal accuracy even in closed loop, given that the noise properties are correctly modeled. It can also be applied to system with arbitrary feedback mechanisms. The direct method should therefore be seen as the first choice of methods for closed-loop identification.

The indirect method requires perfect knowledge of the feedback to give correct results. In case the true controller deviates from the assumed one the results can be arbitrarily bad.

The projection method may be applied to arbitrary closed-loop systems and gives consistent estimates regardless of the nature of the feedback and the noise model used. Thus the noise model can be used as a design variable to tune the frequency weighting in the identification criterion; the price we pay is an increased variance error. For linear systems this method coincides with the two-stage method.

\section{References}

[1] H. Akaike. Some Problems in the Application of the Cross-Spectral Method. In B. Harris, editor, Spectral Analysis of Time Series, pages 81-107. John Wiley \& Sons, 1967.

[2] U. Forssell. Properties and Usage of Closed-loop Identification Methods. Licentiate thesis LIUTEK-LIC-1997:42, Department of Electrical Engineering, Linköping University, Linköping, Sweden, September 1997.

[3] I. Gustavsson, L. Ljung, and T. Söderström. Identification of Processes in Closed Loop - Identifiability and Accuracy Aspects. Automatica, 13:5975,1977 .

[4] L. Ljung. System Identification: Theory for the User. Prentice-Hall, 1987.

[5] P. M. J. Van den Hof and R. J. P. Schrama. An Indirect Method for Transfer Function Estimation from Closed Loop Data. Automatica, 29(6):1523$1527,1993$.

[6] G. Vandersteen. Identification of Linear and Nonlinear Systems in an Errors-in-Variables Least Squares and Total Least Squares Framework. Phd thesis, Vrije Universiteit Brussel, Brussels, Belgium, 1997. 